\title{
Strange Star Equation of State With a Modified Richardson Potential.
}

\author{
Manjari Bagchi ${ }^{\mathrm{a}, 1}$, Subharthi Ray ${ }^{\mathrm{b}}$, Mira Dey ${ }^{\mathrm{a}, 1}$, Jishnu Dey ${ }^{\mathrm{a}, 1}$ \\ a Presidency College, 86/1 College Street, Kolkata 700073, India \\ ${ }^{\mathrm{b}}$ IUCAA, Post bag 4, Ganeshkhind, Pune 411007, India
}

\begin{abstract}
Richardson potential is an phenomenological interquark interaction taking care of two aspects of QCD, namely the asymptotic freedom and the confinement. The original potential has a scale parameter having value $\sim 400 \mathrm{MeV}$ and is well tested in hadronic property calculations. This potential was then used in strange star calculation. Strange stars are very compact stars composed of strange quark matter i.e. a very high density strange quark phase consisting of deconfined $\mathrm{u}, \mathrm{d}$ and $\mathrm{s}$ quarks. Here the value of the scale parameter was taken as $100 \mathrm{MeV}$. The argument was that for a deconfined quark system like a strange star, the scale parameter may have a value quite different from that used in hadronic sector. To remove this discrepancy we introduced two scale parameters in the potential, one for the asymptotic freedom part and the other for the confining part. With suitable values of the parameters, this modified potential has been successfully used in both baryonic property and strange star calculations. The Equation of States obtained with the modified potential are also used to obtain mass-radius relations for the strange stars.
\end{abstract}

Key words: strange stars, compact objects, quark matter PACS: 04.40.Dg, 97.10.Nf, 97.10.Pg, 11.15.Pg, 11.30.Rd

\section{Introduction}

Two decades have passed since the existence of strange quark matter (SQM) and strange stars (SS) were proposed by Witten (1984), and even today it is still difficult to prove or disprove the existence of SS with certainty. But some recent observational signatures in X-ray and $\gamma$-ray astronomy support the

Email address: mnj2003@vsnl.net (Manjari Bagchi).

1 Work supported in part by DST grant no. SP/S2/K-03/2001, Govt. of India. 
existence of SS (Oaknin \& Zhitnitskv, 2005; Bignami et al., 2004; Sinha et al., 2003) inspiring us to produce some new equation of states (EOS) for SQM. Present work is an extension and improvement of the Realistic Strange Star Model Dev et al. (1998).

\section{Realistic Strange Star (ReSS) Model and its modification}

Using the inverse of number of colors as an expansion parameter in QCD ('t Hooft, 1974), baryon like systems as SS can be explored in relativistic tree level calculation with an average interquark potential. Richardson potential is a phenomenological one used for this purpose. The original potential has the asymptotic freedom (AF) and confinement built in (Richardson, 1979) and is used successfully in mesonic (Crater \& van Alstine, 1984) and baryonic (Dev et al., 1986) property calculations. The potential is :

$$
V_{i j}=\frac{12 \pi}{27} \frac{1}{\ln \left(1+\left(\mathbf{k}_{i}-\mathbf{k}_{j}\right)^{2} / \Lambda^{2}\right)} \frac{1}{\left(\mathbf{k}_{i}-\mathbf{k}_{j}\right)^{2}}
$$

$\left(\mathbf{k}_{i}-\mathbf{k}_{j}\right)$ being the momentum transfer. The scale parameter $\Lambda$ is $\sim 400 \mathrm{MeV}$ for hadron phenomenology. But in SS calculation $\Lambda$ is taken $\sim 100 \mathrm{MeV}$. This discrepancy ultimately leads us to modify the potential. The potential is screened due to gluon propagation in a medium and $\left(\mathbf{k}_{i}-\mathbf{k}_{j}\right)^{2}$ is replaced by $\left[\left(\mathbf{k}_{i}-\mathbf{k}_{j}\right)^{2}+D^{-2}\right]$. Inverse screening length $D^{-1}$ (i.e. gluon mass $m_{g}$ ) is (Dev et al., 1998):

$$
\left(D^{-1}\right)^{2}=\frac{2 \alpha_{0}}{\pi} \sum_{i=u, d, s,} k_{i}^{f} \sqrt{\left(k_{i}^{f}\right)^{2}+M_{i}^{2}}
$$

The Fermi momentum $\left(k_{i}^{f}\right)$ is related to the corresponding number density:

$$
k_{i}^{f}=\left(n_{i} \pi^{2}\right)^{1 / 3}
$$

Perturbative quark gluon coupling, $\alpha_{0}$ has value $\sim 0.2$. The quark mass, $M_{i}$ is taken to be density dependent to restore chiral symmetry at high density :

$$
M_{i}=m_{i}+M_{Q} \operatorname{sech}\left(\frac{n_{B}}{N n_{0}}\right), \quad i=u, d, s .
$$

where the constituent quark mass $M_{Q}$ lies between 300-350 $\mathrm{MeV}$ for each quark, $n_{B}=\left(n_{u}+n_{d}+n_{s}\right) / 3$ is the baryon number density, $n_{0}$ is the normal nuclear matter density and $N$ is a parameter. The current quark masses, $m_{i}$ are taken as 4, 7 and $150 \mathrm{MeV}$ for u, d, s quarks respectively. From the charge neutrality and beta equlibrium conditions, $k_{i}^{f}$ and $\mu_{i}$ (chemical potentials) of quarks and electron are obtained as a function of $n_{B}$ and performing a relativistic Hartree-Fock calculation, the energy density $\epsilon$ of the SQM is obtained. 
The first law of thermodynamics gives the EOS of SQM at zero temperature as:

$$
P=\sum_{i}\left(\mu_{i} n_{i}-\epsilon_{i}\right)
$$

To obtain a realistic EOS, the model parameters $\alpha_{0}, M_{Q}$ and $N$ are chosen in such a way that the minimum value of energy per baryon $\left(E / A \equiv \epsilon / n_{B}\right)$ for uds quark matter is less than that of the most stable element $F e^{56}$ i.e. 930 $M e V$. Thus uds quark matter can construct stable stars. The minimum value of $E / A$ is obtained at the star surface where the pressure is zero. The presence of zero pressure indicates the existence of a sharp surface of the strange star in contrast to fuzzy surfaces of the neutron stars. The surface is sharp since strong interaction dictates the deconfinement point. On the contrary; for the same parameters, the minimum value of $E / A$ for ud quark matter is greater than that of $F e^{56}$ so that $F e^{56}$ remains the most stable element in the nonstrange world. The EOS for strange quark matter is now used to get the Mass-Radius configuration of the strange star by solving the TOV equations with appropriate boundary conditions.

In the present work, we have assumed that the scales for the two phenomena $\mathrm{AF}$ and confinement are different and the modified Richardson potential is:

$$
V_{i j}=\frac{12 \pi}{27}\left[\frac{1}{\left(\mathbf{k}_{i}-\mathbf{k}_{j}\right)^{2} \ln \left(1+\frac{\left(\mathbf{k}_{i}-\mathbf{k}_{j}\right)^{2}}{\Lambda^{2}}\right)}-\frac{\Lambda^{2}}{\left(\mathbf{k}_{i}-\mathbf{k}_{j}\right)^{4}}+\frac{\Lambda^{\prime 2}}{\left(\mathbf{k}_{i}-\mathbf{k}_{j}\right)^{4}}\right]
$$

Here $\Lambda$ is the scale representing the AF as the first two terms are asymptotically zero for large $\left(\mathbf{k}_{i}-\mathbf{k}_{j}\right) . \Lambda^{\prime}$ is the scale corresponding to confinement as the third term reduces to a linear confinement for small $\left(\mathbf{k}_{i}-\mathbf{k}_{j}\right)$. From baryonic property calculations (Bagchi et al., 2004), we have found the appropriate values of the parameters as $\Lambda=100 \mathrm{MeV}$ and $\Lambda^{\prime}=350 \mathrm{MeV}$. So we have applied the modified potential with these values of the parameters in SS calculations.

\section{Results}

We have obtained different EOSs for different set of parameters (see table 1). Though baryonic property calculation tells that $\Lambda^{\prime}$ should be $350 \mathrm{MeV}$, we have determined EOSs (C, D, E) also for $\Lambda^{\prime}=300 \mathrm{MeV}$ only for theoretical interest i.e. to see the effect of changing other parameters of the model. The other $\operatorname{EOSs}\left(\mathrm{A}, \mathrm{B}, \mathrm{F}\right.$ ) are indeed for $\Lambda^{\prime}=350 \mathrm{MeV}$. Comparing with Dev et al. (1998), it is clear that the nature of EOSs and M - R relations do not change appreciably by the use of the modified potential if we adjust other 
Table 1

Different EOSs using the modified potential. $\Lambda$ is always $100 \mathrm{MeV} . \epsilon_{c} / \mathrm{c}^{2}$ is the central density. The present value of $\alpha_{0}$ is 0.55-0.65 as compared to 0.20 of Dev et al. (1998). We have to chose a larger value of $\alpha_{0}$ as the confinement parameter has larger value. Such a large value of $\alpha_{0}$ is in well agreement with theoretical results (Rav et al., 2000).

\begin{tabular}{|c|c|c|c|c|c|c|c|c|c|}
\hline EOS & $\Lambda^{\prime}$ & $M_{q}$ & $N$ & $\alpha_{0}$ & $\left(\frac{E}{A}\right)_{\min }^{u d s}$ & $\left(\frac{E}{A}\right)_{\min }^{u d}$ & $M_{\max }$ & $\begin{array}{c}\mathrm{R} \\
\text { for } \\
M_{\max }\end{array}$ & $\begin{array}{c}\epsilon_{c} / c^{2} \\
\text { for } M_{\max } \\
\end{array}$ \\
& $M e V$ & $M e V$ & & & $M e V$ & $M e V$ & $M_{\odot}$ & $k m$ & $g m c^{14}$ \\
\hline \hline $\mathrm{A}$ & 350 & 325 & 3.0 & .55 & 874 & 942 & 1.53 & 7.41 & 42.98 \\
\hline $\mathrm{B}$ & 350 & 325 & 3.0 & .65 & 907 & 942 & 1.47 & 7.14 & 45.48 \\
\hline $\mathrm{C}$ & 300 & 325 & 2.7 & .55 & 877 & 942 & 1.55 & 7.53 & 41.46 \\
\hline $\mathrm{D}$ & 300 & 325 & 3.0 & .55 & 906 & 950 & 1.46 & 7.11 & 46.14 \\
\hline $\mathrm{E}$ & 300 & 335 & 3.0 & .55 & 912 & 973 & 1.45 & 7.02 & 47.65 \\
\hline $\mathrm{F}$ & 350 & 345 & 3.0 & .65 & 920 & 987 & 1.44 & 6.97 & 48.07 \\
\hline \hline
\end{tabular}

model parameters suitably; but the value of $m_{g}$ is significantly larger as we have to increase the value of $\alpha_{0}$ to counter balance the increase in $\Lambda^{\prime}$. The compactness $(\mathrm{M} / \mathrm{R}$ ratio) of the stars have increased for the new EOSs than that obtained by Dev et al. (1998). Zero pressure is obtained for $n_{B}>4 n_{0}$ for all EOSs implying that in a strange star, $n_{B}$ can not be less than $4 n_{0}$. The central density may have different values giving rise to stars of different masses, but for standard masses i.e. around 1.4-1.5 $M_{\odot}$, it becomes $\sim 13 n_{0}$. This high density indicates a high pressure.

\section{References}

Bagchi, M., Dey, M., Daw, S., Dey, J., A model finding a new Richardson potential with different scales for confinement and asymptotic freedom, by fitting the properties of $\Delta^{++}$and $\Omega^{-}, 2004$, Nucl. Phys. A, 740, 109-118.

Bignami, G. F., Luca, A. D., Caraveo, P. A., et al., 1E1207.4-5209 - a unique object, astro-ph/0402231.

Crater, H. W., van Alstine, P., Relativistic naive quark model for spinning quarks in mesons, 1984, Phys. Rev. Lett., 53, 1527-1530.

Dey, M., Bombaci, I., Dey, J., Ray, S., Samanta, B. C., Strange stars with realistic quark vector interaction and phenomenological density dependent 
scalar potential, 1998, Phys. Lett. B, 438, 123-128.

Dey, J., Dey, M., Tourneux, J. L., QCD-based relativistic Hartree-Fock calculations for identical quarks, 1986, Phys. Rev. D, 34, 2104-2111.

Hooft, G. 't, A planar diagram theory for strong interactions, 1974, Nucl. Phys. B, 72, 461-473.

Oaknin, D. H., Zhitnitsky, A. R., $511 \mathrm{keV}$ photons from color superconducting dark matter, 2005, Phys. Rev. Lett., 94, 101301.

Ray, S., Dey, J., Dey, M., Density dependent strong coupling constant of QCD derived from compact star data, 2000, Mod. Phys. Lett. A, 15, 1301-1306.

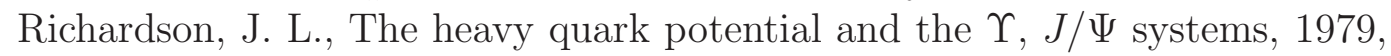
Phys. Lett. B, 82, 272-274.

Sinha, M., Dey, J., Dey, M., Ray, S., Bhowmick, S., Have we observed the skin vibration of realistic strange stars (ReSS)?, 2003, Mod. Phys. Lett. A, 18, 661-667.

Witten, E., Cosmic separation of phases, 1984, Phys. Rev. D., 30, 272-285. 